\title{
Decreased muscle strength is associated with proinflammatory cytokines but not testosterone levels in men with diabetes
}

\author{
J.P. Ferreira ${ }^{1}$, A.M.O. Leal ${ }^{2}$, F.A. Vasilceac ${ }^{3}$, C.D. Sartor ${ }^{4,5}$, I.C.N. Sacco ${ }^{4}$, A.S. Soares ${ }^{1}$ and \\ T.F. Salvini ${ }^{1}$ \\ ${ }^{1}$ Laboratório de Plasticidade Muscular, Departamento de Fisioterapia, Universidade Federal de São Carlos, São Carlos, SP, Brasil \\ ${ }^{2}$ Departamento de Medicina, Universidade Federal de São Carlos, São Carlos, SP, Brasil \\ ${ }^{3}$ Departamento de Gerontologia, Universidade Federal de São Carlos, São Carlos, SP, Brasil \\ ${ }^{4}$ Departamento de Fisioterapia, Fonoaudiologia e Terapia Ocupacional, Faculdade de Medicina, Universidade de São Paulo, \\ São Paulo, SP, Brasil \\ ${ }^{5}$ Departamento de Fisioterapia, Universidade Ibirapuera, São Paulo, SP, Brasil
}

\begin{abstract}
The aim of this study was to compare muscle strength in male subjects with type 2 diabetes mellitus (DM2) with and without low plasma testosterone levels and assess the relationship between muscle strength, testosterone levels, and proinflammatory cytokines. Males (75) aged between 18 and 65 years were divided into 3 groups: control group that did not have diabetes and had a normal testosterone plasma level ( $>250 \mathrm{ng} / \mathrm{dL}$ ), DnormalTT group that had DM2 with normal testosterone levels, and the DlowTT group that had DM2 and low plasma testosterone levels $(<250 \mathrm{ng} / \mathrm{dL}$ ). The age (means \pm SD) of the groups was $48.4 \pm 10,52.6 \pm 7$, and $54.6 \pm 7$ years, respectively. Isokinetic concentric and isometric torque of knee flexors and extensors were analyzed by an isokinetic dynamometer. Plasma testosterone and proinflammatory cytokine levels were determined by chemiluminescence and ELISA, respectively. Glycemic control was analyzed by glycated hemoglobin (HbA1C). In general, concentric and isometric torques were lower and tumor necrosis factor (TNF)- $\alpha$, interleukin (IL)- 6 , and IL-1 $\beta$ plasma levels were higher in the groups with diabetes than in controls. There was no correlation between testosterone level and knee torques or proinflammatory cytokines. Concentric and isometric knee flexion and extension torque were negatively correlated with TNF- $\alpha$, IL-6, and HbA1C. IL-6 and TNF- $\alpha$ were positively correlated with HbA1C. The results of this study demonstrated that muscle strength was not associated with testosterone levels in men with DM2. Low muscle strength was associated with inflammatory markers and poor glycemic control.
\end{abstract}

Key words: Diabetes; Testosterone; Muscle strength; Cytokines

\section{Introduction}

Clinical and epidemiological evidence demonstrates that men with type 2 diabetes mellitus (DM2), metabolic syndrome, and obesity exhibit low plasma testosterone levels (1). Low testosterone levels are associated with metabolic and cardiovascular complications, sexual dysfunction, risk of bone fracture, and reduced muscle strength (2).

Around $20 \%$ of people with DM2 show a decline in testosterone levels (3) from disease onset (2). The causal interactions between obesity, metabolic syndrome, DM2, and testosterone deficiency are complex. In short, increased activity of the aromatase enzyme in adipose tissue raises estradiol levels, which inhibits the hypothalamic-pituitaryadrenal axis and prompts a decline in testicular production of testosterone. Additionally, hormones (leptin) and inflammatory mediators such as interleukin (IL)-1, IL-6, and tumor necrosis factor (TNF)- $\alpha$ in adipose tissue can also compromise testicular function (4).

Testosterone replacement therapy (TRT) can improve libido, sexual function, bone density, muscle mass preservation, body composition, mood, erythropoiesis, cognition, and quality of life as well as can lower the risk of cardiovascular disease $(5)$ in obese men with DM2 $(5,6)$. However, the topic is controversial because TRT is associated with increased risk of prostate cancer by worsening symptoms of benign prostatic hypertrophy, liver toxicity, hyperviscosity, erythrocytosis, severe heart failure, and cardiovascular disease, and exacerbates untreated sleep apnea, as previously reviewed (7-9). Thus, physicians should

Correspondence: T.F. Salvini: <tania@ufscar.br>

Received February 3, 2017 | Accepted May 11, 2018 
discuss symptom severity with the patients, including those resulting from loss of muscle strength, and weigh the potential risks and benefits of TRT $(5,6)$.

Regardless of other factors, people with low testosterone levels develop reduced muscle strength (10). This is because testosterone stimulates protein synthesis and the recruitment of satellite cells (11). Additionally, testosterone inhibits production of the inflammatory cytokines IL-1 and IL-6 (12), which are known to activate apoptosis pathways and muscle atrophy (13). However, there is no study that analyzed the muscle strength of people with DM2 and the disease relationship to plasma testosterone levels. Although some research has shown that individuals with DM2 exhibit reduced muscle strength (14), whether low testosterone levels can exacerbate loss of strength in these subjects has yet to be established. Isokinetic dynamometry is a safe, reliable, and reproducible method to assess joint torque, providing the strength of individuals under different types of contraction (15). In addition, knee muscle strength plays an important role in movement and quality of life.

Therefore, this study aimed to evaluate the concentric and isometric torque of knee flexion and extension in diabetic men with and without hypogonadism. The presence of subclinical inflammation in DM2 and its influence on the musculoskeletal system is well known, activating pathways of apoptosis muscle atrophy (13) and reducing testicular testosterone secretion (16). As such, the present investigation analyzed participants' plasma levels of the proinflammatory cytokines IL-1, IL-6, and TNF- $\alpha$.

Given that its association with subclinical inflammation means that DM2 can affect muscle strength (17), and that hypogonadism can also affect muscle strength (18) independently of DM2, the hypothesis of the study was that knee flexion and extension strength is lower in subjects with both DM2 and hypogonadism compared to subjects with DM2 and control subjects without hypogonadism.

\section{Material and Methods}

\section{Subjects}

The participants were recruited from local healthcare units and the Endocrinology Clinic of the Federal University of São Carlos. Males aged between 18 and 65 years were included in the study. The inclusion criteria for the control group were men without DM2 (19) and with normal total testosterone levels (20), for the DnormalTT group, men with DM2 (19) and normal total testosterone levels, and for the DlowTT group, men with DM2 (19) and low total testosterone levels (20). A total of 287 subjects were interviewed for eligibility. The exclusion criteria were cardiac diseases, pulmonary emphysema, knee arthrosis or arthritis, a history of knee ligament or meniscus injuries, herniated discs, stroke, peripheral diabetic neuropathy (PDN), and anti-inflammatory or hormonal therapy. The 75 subjects who met the inclusion criteria were distributed into the three groups: Control $(n=20)$, DnormalTT $(n=45)$, and DlowTT $(n=10)$ (Figure 1).

The study complied with the Guidelines and Regulations for Research Involving Human Subjects (National Health Council Resolution 196/1996) and was approved by the university's Research Ethics committee (protocol No. 797.125). All participants took part voluntarily and gave written informed consent.

\section{Clinical evaluation}

PDN was evaluated by a trained physical therapist using the following clinical parameters: i) typical neuropathy symptoms assessed by a questionnaire based on the Michigan Neuropathy Screening Instrument (21); ii) tactile sensitivity using a $10 \mathrm{~g}$ Semmes-Weinstein monofilament (Sorri-Baru, Brazil) tested in four plantar areas (hallux plantar face, and the 1st, 3rd, and 5th metatarsals); and iii) vibratory perception with a $128 \mathrm{~Hz}$ tuning fork applied to the medial region of the hallux interphalangeal joint (21). To determine the presence of PDN, these variables were processed in artificial intelligence Fuzzy Logic System software (LaBiMPH, Brazil), described in greater details in previous studies $(22,23)$. The software combines each fuzzy set of the input variables and gives the degree of PDN between 0 and 10, classified as follows: i) $<2.5$ absent; ii) 2.5-5.0 mild; iii) 5.1-8.0 moderate; iv) $>8.0$ severe (22). The degree obtained by the fuzzy model showed a very strong correlation with the expert's assessment (Pearson's coefficient $r=0.943$ ) and a high level of accuracy when classifying real patients analyzed with the model (ROC curve area=0.91) (22).

\section{Anthropometric and glycemic control data}

Body mass index (BMI) was calculated as follows: weight $(\mathrm{kg}) /$ height $^{2}(\mathrm{~cm})$. The glycated hemoglobin $(\mathrm{HbA} 1 \mathrm{c})$ percentage is a 3-month indicator of glucose control and was measured in all participants (19).

\section{Plasma testosterone}

Total plasma testosterone level was determined by chemiluminescence (24). Data are reported in $\mathrm{ng} / \mathrm{dL}$ and total testosterone $<250 \mathrm{ng} / \mathrm{dL}$ was considered low (20).

\section{Proinflammatory cytokines}

Plasma concentrations of TNF- $\alpha, \mathrm{IL}-1 \beta$, and IL- 6 were analyzed by sandwich ELISA (enzyme-linked immunosorbent assay) and each cytokine was tested according to the manufacturer's instructions (25). Readings were performed using a 490-nm filter. The detection limits of the cytokines in the serum were $5 \mathrm{pg} / \mathrm{mL}$ for $\mathrm{IL}-1 \beta, 2.62 \mathrm{pg} / \mathrm{mL}$ for IL-6, and $1.7 \mathrm{pg} / \mathrm{mL}$ for TNF- $\alpha$. After analyses, the data were transformed and normalized by the standard curve.

\section{Torque analysis}

Concentric and isometric torque of knee flexors and extensors were analyzed using an isokinetic dynamometer 


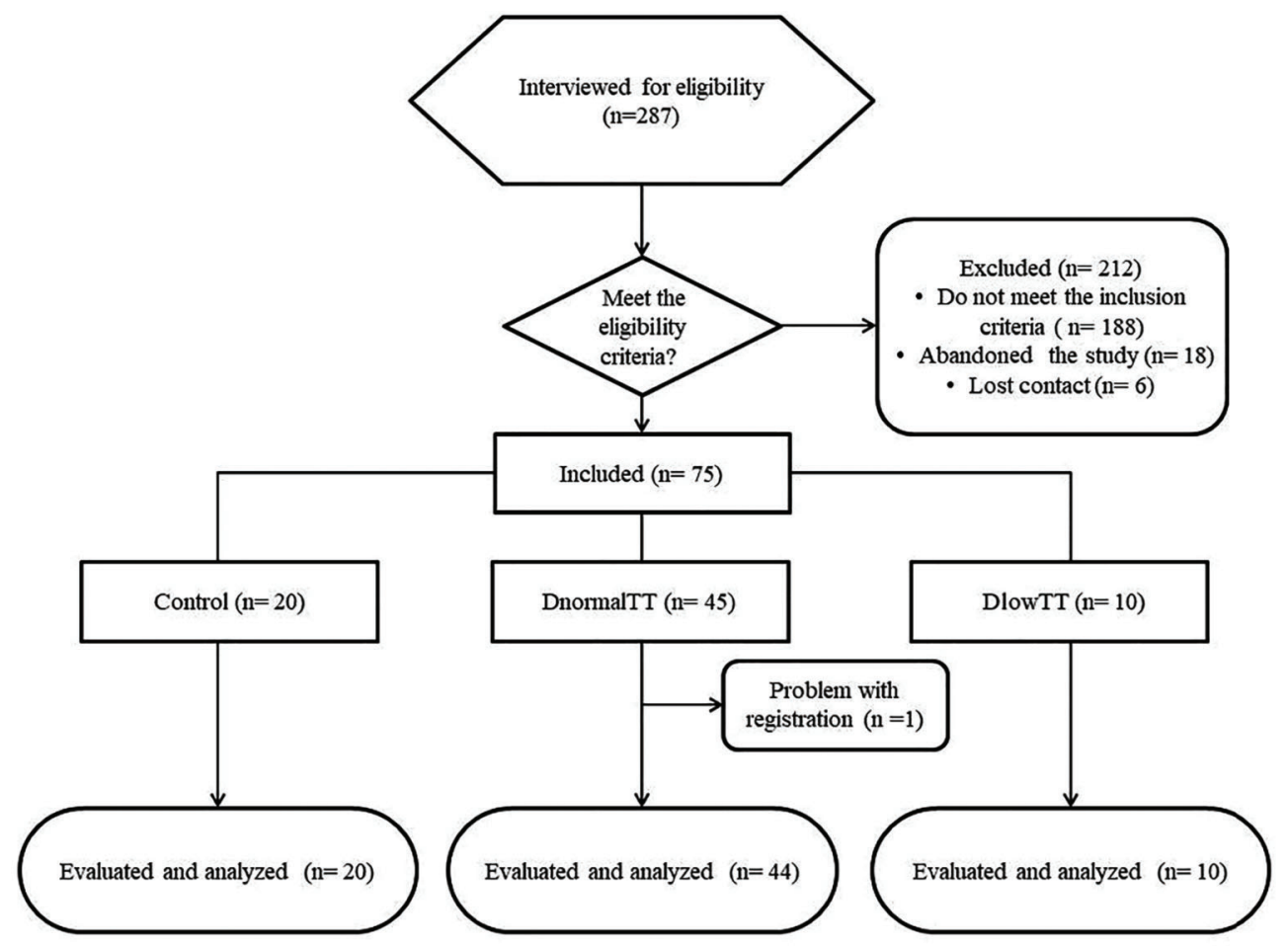

Figure 1. Flowchart of the study design. Control: control group; DnormalTT: diabetics with normal total testosterone; DlowTT: diabetics with low total testosterone.

(Biodex, System III, USA). Concentric torque was analyzed at $60 \%$ s. The equipment was calibrated according to manufacturer's recommendations before the assessments. Individuals were asked which leg they could kick a ball hardest in order to determine their dominant limb. The type of contraction tested first was chosen randomly using a randomization spreadsheet. Before maximal testing, submaximal familiarization sessions were conducted with 3 repetitions for each movement in the concentric mode and 2 repetitions in the isometric mode. A 1.5-min rest was allowed between modes and 3 min between familiarization and the maximal test. During the test, participants remained seated in the dynamometer chair with the backrest at $85^{\circ}$, trunk stabilized and fixed to the backrest using two belts with an additional pelvic belt. The axis of rotation of the dynamometer was aligned with the lateral epicondyle of the femur and the resistance arm fixed to the distal third of the leg, just above the malleolus.

Verbal and visual encouragement was provided during all maximal voluntary contractions, always by the same evaluator. After the analyses, the text files of the tests were processed in MATLAB (version 7.0.1, MathWorks, USA) to determine the peak torque achieved by participants in the repetitions. Peak torque in $\mathrm{N}-\mathrm{m}$ (newton meters) was normalized by the weight of the individual and multiplied by 100 .

\section{Statistical analysis}

The Levene and the Kolmogorov-Smirnov tests were applied to determine homogeneity of variance and normal distribution, respectively. One-way ANOVA and Tukey's post-hoc test were performed to compare the clinical and demographic characteristics of the groups, as well as concentric and isometric torque of the knee flexors and extensors. Significance was set at 5\%. Effect sizes (Hedges' g) of torque values between groups were also calculated, and considered insignificant (0.00-0.19), small (0.20-0.39), medium (0.40-0.79), or large $(\geqslant 0.80)(26)$.

Kruskal-Wallis and Mann-Whitney $U$ tests were applied to analyze the proinflammatory cytokines. For nonparametric comparisons, the significance level was adjusted according to the number of comparisons and set at $\mathrm{P} \leqslant$ 0.016 . Correlations between concentric and isometric knee flexion and extension torque, proinflammatory cytokines, and $\mathrm{HbA} 1 \mathrm{c}$ were assessed using Pearson's correlation coefficient ( $r=0.10-0.29$ : low correlation; $r=0.30-0.49$ : medium correlation; $r=0.50-1$ : high correlation) (27).

\section{Results}

One individual in the DnormalTT group was unable to complete the test. All groups were similar in terms of age. The DlowTT group had a higher BMI than the control 
Table 1. Clinical characteristics of the participants.

\begin{tabular}{|c|c|c|c|c|}
\hline & Control $(n=20)$ & DnormalTT $(n=44)$ & DlowTT $(n=10)$ & ANOVA \\
\hline Age (years) & $48.40(10.03)$ & $52.61(7.81)$ & $54.60(7.24)$ & $F=2.39 ; P=0.09$ \\
\hline Time since diagnosis (months) & $0.0(0)$ & $108.90(72.48)^{\star}$ & $89.40(75.17)^{\star}$ & $F=21.15 ; P=0.00$ \\
\hline Testosterone (ng/dL) & $402.0(292.7)$ & $369.2(84.2)$ & $204.6(44.2)^{\star \#}$ & $F=0.60 ; P=0.55$ \\
\hline $\mathrm{HbA} 1 \mathrm{C}(\%)$ & $5.3(0.4)$ & $8.5(2.5)^{*}$ & $9.1(2.2)^{*}$ & $F=2.74 ; P=0.07$ \\
\hline BMI $\left(\mathrm{kg} / \mathrm{m}^{2}\right)$ & $26.4(4.1)$ & $27.8(3.1)$ & $30.7(5.0)^{*}$ & $F=4.42 ; P=0.01$ \\
\hline Degree of peripheral neuropathy (Fuzzy score) & $0.67(0.2)$ & $1.2(0.9)^{*}$ & $0.8(0.2)$ & $F=4.39 ; P=0.01$ \\
\hline Oral antidiabetic / insulin / oral (n) & & $39 / 0 / 5$ & $9 / 0 / 1$ & \\
\hline
\end{tabular}

Data are reported as means \pm SD. DnormalTT: type 2 diabetics with normal total testosterone; DlowTT: type 2 diabetics with low total testosterone. ${ }^{*} \mathrm{P}<0.05$ compared to controls; ${ }^{\#} \mathrm{P}<0.05$ compared to DnormalTT.

Table 2. Peak torque for the different contraction types.

\begin{tabular}{llccccccc}
\hline $\begin{array}{l}\text { Type of } \\
\text { Contraction }\end{array}$ & $\begin{array}{c}\text { Joint } \\
\text { movement }\end{array}$ & $\begin{array}{c}\text { Control } \\
(\mathrm{n}=20)\end{array}$ & $\begin{array}{c}\text { Effect size } \\
\text { Control vs } \\
\text { DnormalTT }\end{array}$ & $\begin{array}{c}\text { DnormalTT } \\
(\mathrm{n}=44)\end{array}$ & $\begin{array}{c}\text { Effect size } \\
\text { DnormalTT } \\
\text { vs DlowTT }\end{array}$ & $\begin{array}{c}\text { DlowTT } \\
(\mathrm{n}=10)\end{array}$ & $\begin{array}{c}\text { Effect size } \\
\text { Control vs } \\
\text { DlowTT }\end{array}$ & $\begin{array}{c}\text { ANOVA } \\
\text { Concentric }\end{array}$ \\
& Flexion & $94.18(33.87)$ & -1.58 & $53.35(21.16)^{*}$ & 0.59 & $66.67(28.37)^{*}$ & 0.85 & $\mathrm{~F}=16.8 ; \mathrm{P}=0.00$ \\
& Extension & $156.61(56.50)$ & -1.17 & $104.36(37.68)^{*}$ & 0.74 & $132.98(41.79)$ & 0.45 & $\mathrm{~F}=10.0 ; \mathrm{P}=0.00$ \\
Isometric & Flexion & $99.65(35.77)$ & -0.65 & $80.46(25.99)$ & -0.33 & $70.81(40.73)^{*}$ & 0.77 & $\mathrm{~F}=3.73 ; \mathrm{P}=0.02$ \\
& Extension & $218.90(54.04)$ & -0.81 & $171.95(58.88)^{*}$ & 0.17 & $181.65(43.10)$ & 0.73 & $\mathrm{~F}=4.89 ; \mathrm{P}=0.01$ \\
\hline
\end{tabular}

Peak torque is reported as means \pm SD $(\mathrm{N} . \mathrm{m} / \mathrm{kg} \times 100)$. DnormalTT: type 2 diabetics with normal total testosterone; DlowTT: type 2 diabetics with low total testosterone. Effect size: insignificant (0.00-0.19), small (0.20-039), medium $(0.40-0.79)$, large ( $\geqslant 0.80)$. ${ }^{*} \mathrm{P}<0.05$ compared to controls.

Table 3. Comparison of inflammatory markers tumor necrosis factor (TNF- $\alpha$ ), interleukin- 6 (IL-6), interleukin 1- beta (IL-1 $\beta$ ).

\begin{tabular}{lcccc}
\hline & Control $(\mathrm{n}=20)$ & DnormalTT $(\mathrm{n}=44)$ & DlowTT $(\mathrm{n}=10)$ & Kruskal-Wallis test \\
\hline TNF $\alpha(\mathrm{pg} / \mathrm{mL})$ & $0.71(0.71-0.71)$ & $0.74(0.71-1.00)^{*}$ & $1.00(0.74-1.00)^{*}$ & $\mathrm{H}=46.2 ; \mathrm{P}<0.01$ \\
$\mathrm{IL}-6(\mathrm{pg} / \mathrm{mL})$ & $0.50(0.51-0.51)$ & $0.73(0.51-0.83)^{*}$ & $0.73(0.73-0.83)^{*}$ & $\mathrm{H}=45.0 ; \mathrm{P}<0.01$ \\
$\mathrm{IL}-1 \beta(\mathrm{pg} / \mathrm{mL})$ & $0.87(0.87-0.87)$ & $0.90(0.84-0.90)^{*}$ & $0.90(0.84-0.90)^{*}$ & $\mathrm{H}=14.0 ; \mathrm{P}<0.01$ \\
\hline
\end{tabular}

Data are reported as means (minimum-maximum). DnormalTT: type 2 diabetics with normal total testosterone; DlowTT: type 2 diabetics with low total testosterone. ${ }^{*} \mathrm{P}<0.016$ compared to controls.

group $(P \leqslant 0.01)$; however, there was no difference in $\mathrm{BMI}$ between the two groups with DM2. Intergroup differences were observed for $\mathrm{HbA} 1 \mathrm{C}$ values, but only between the groups with DM2 and the control group $(P<0.01$; Table 1).

Peak torque was similar for all movements and both contraction types in the groups with DM2. When compared to controls, the DnormalTT group showed lower concentric and isometric knee extension torques $(P<0.01)$ and the DlowTT exhibited lower isometric knee flexion torque $(P=0.04)$. Both groups with DM2 showed lower concentric knee flexion torque than controls $(\mathrm{P}<0.02$; Table 2$)$.

TNF- $\alpha$, IL-6, and IL-1 $\beta$ concentrations were higher in the groups with DM2 than controls $(P<0.01)$, but no difference was found between the DnormalTT and DlowTT groups (Table 3). HbA1c was positively correlated with IL-6 and TNF- $\alpha(r=0.55, P<0.01 ; r=0.59, P<0.01)$ and negatively correlated with concentric knee flexion and extension torque $(r=-0.30, P<0.01 ; r=-0.24, P=0.03)$. Concentric and isometric knee flexion and extension torque were negatively correlated with TNF- $\alpha(r=-0.27, \mathrm{P}<0.01$; $r=-0.33, P<0.01 ; \quad r=-0.35, P<0.01 ; r=-0.43, P<0.01$, respectively, for TNF- $\alpha$ vs concentric knee flexion; TNF- $\alpha$ vs concentric knee extension; TNF- $\alpha$ vs isometric knee flexion, and TNF- $\alpha$ vs isometric knee extension) and IL-6 $(r=-0.22, P=0.03 ; r=-0.27, P<0.01 ; r=-0.38, P<0.01$; $r=-0.41, P<0.01$, respectively, for IL-6 vs concentric knee flexion; IL-6 vs concentric knee extension; IL-6 vs isometric knee flexion, and IL-6 vs isometric knee extension). 
There was no correlation between testosterone level and knee torques, or proinflammatory cytokines, considering all groups.

\section{Discussion}

The results of the present study showed reduced isometric and concentric torque in individuals with DM2 regardless of testosterone levels and their association with high IL- 6 and TNF- $\alpha$ concentrations. These findings were consistent with the important pathophysiological role of inflammation in reducing muscle strength.

With respect to decreased muscle strength in individuals with DM2, our results confirmed the findings of previous studies showing that individuals with diabetes have lower skeletal muscle strength than those without diabetes $(28,29)$. The mechanism of muscle strength decline in DM2 subjects is not well defined. In the present study, clinical neuropathy was excluded, but subclinical neuropathy could not be excluded.

We hypothesized that low testosterone levels would negatively influence decreased muscle strength in individuals with DM2. However, both diabetic groups (normal and low testosterone subgroup) exhibited lower muscle strength than control subjects and there was no correlation between testosterone levels and muscle strength. Testosterone administration has been associated with increased muscle strength. However, previous studies $(10,30)$ that examined the correlation between endogenous testosterone and muscle strength have been inconclusive, possibly because circulating testosterone levels may not directly or linearly reflect its biological action on target tissues.

Mechanistically, the interaction between low muscle strength and high $\mathrm{HbA} 1 \mathrm{c}$ may be explained by the effect of hyperglycemia on skeletal muscle mitochondrial dysfunction, protein degradation, and autophagy pathways $(31,32)$, as well as the accumulation of advanced glycation products and oxidative stress (33).

Another potential mechanism for decreased muscle strength in individuals with DM2 is the rise in inflammatory cytokine levels. In the present study, the inflammatory markers TNF- $\alpha, \mathrm{IL}-6$, and IL- $1 \beta$ concentrations were higher in both groups with DM2 than in controls. It has

\section{References}

1. Grossmann M. Low testosterone in men with type 2 diabetes: significance and treatment. J Clin Endocrinol Metab 2011; 96: 2341-2353, doi: 10.1210/jc.2011-0118.

2. Cheung KKT, Luk AOY, So WY, Ma RCW, Kong APS, Chow FCC, et al. Testosterone level in men with type 2 diabetes mellitus and related metabolic effects: A review of current evidence. J Diabets Investig 2015; 6: 112-123, doi: 10.1111/ jdi.12288.

3. Al Hayek AA, Robert AA, Alshammari G, Hakami H, Al Dawish MA. Assessment of hypogonadism in men with type 2 diabetes: been previously demonstrated that increased plasma concentration of inflammatory cytokines may cause loss of strength in these individuals $(13,34)$. Inflammatory cytokines may lead to the inhibition of skeletal muscle protein synthesis and myoblast differentiation by activating MAFbx/ atrogin-1, elF3-f, MyoD, and MuRf1 (35-37). In addition, it has been reported that increased TNF- $\alpha$ levels may activate the caspase pathway, leading to skeletal muscle atrophy due to enzymatic fragmentation of muscle DNA $(38,33)$.

In conclusion, the results of this study demonstrated that muscle strength was lower in individuals with DM2 and was not associated with low total testosterone levels. Our results also indicated that reduced muscle strength in subjects with DM2 subjects could be associated with an increase in the inflammatory cytokines IL- 6 and TNF- $\alpha$ and poor glycemic control. However, further studies are needed to clarify this association.

As limitations, the DlowTT group was a very small sample and despite the number of individuals interviewed, only 10 individuals with DM2 and low testosterone met the eligibility criteria of the study. Given the sample size evaluated in the DlowTT group, one-way ANOVA as the statistical design, and an alpha error of $5 \%$, the statistical power $(1-\beta)$ obtained using a small effect size was 0.70 . We also calculated the sample size using a power of 0.93 and medium effect size (0.45). For future studies, we recommend using a sample size of 75 individuals equally distributed among groups. In addition, it would be interesting for future studies to analyze the association between strength and low testosterone level with the quality of life and physical activity level of these individuals.

\section{Acknowledgments}

This study was supported by Fundação de Amparo à Pesquisa do Estado de São Paulo (FAPESP, Process No. 2011/22122-5) and Conselho Nacional de Desenvolvimento Científico e Tecnológico (CNPq). T.F. Salvini, I.C.N. Sacco, and C.D. Sartor are funded by CNPq (Process Nos. 3013442013-2, 305606/2014-0, and 151531/2013-7, respectively). J.S.P. Ferreira received a PhD scholarship from the Coordenação de Aperfeiçoamento de Pessoal de Nível Superior (CAPES) (Process No. 1662695). a cross-sectional study from Saudi Arabia. Clin Med Insights Endocrinol Diabetes 2017; 10: 1179551417710209.

4. Wang C, Jackson G, Jones TH, Matsumoto AM, Nehra A, Perelman MA, et al. Low testosterone associated with obesity and the metabolic syndrome contributes to sexual dysfunction and cardiovascular disease risk in men with type 2 diabetes. Diabetes Care 2011; 34: 1669-1675, doi: 10.2337/dc10-2339.

5. Bassil N, Alkaade S, Morley JE. The benefits and risks of testosterone replacement therapy: a review. Ther Clin Risk Manag 2009; 5: 427-448. 
6. Seftel $A D$, Kathrins M, Niederberger C. Critical update of the 2010 endocrine society clinical practice guidelines for male hypogonadism a systematic analysis. Mayo Clinic Proc 2015; 90: 1104-1115, doi: 10.1016/j.mayocp.2015.06.002.

7. Michaud JE, Billups KL, Partin AW. Testosterone and prostate cancer: an evidence-based review of pathogenesis and oncologic risk. Ther Adv Urol 2015; 7: 378-387, doi: 10.1177/1756287215597633.

8. Onasanya O, lyer G, Lucas E, Lin D, Singh S, Alexander GC. Association between exogenous testosterone and cardiovascular events: an overview of systematic reviews. Lancet Diabetes Endocrinol 2016; 4: 943-956, doi: 10.1016/S22138587(16)30215-7.

9. Allan C a, Strauss BJG, McLachlan RI. Body composition, metabolic syndrome and testosterone in ageing men. Int $\mathrm{J}$ Impot Res. 2007; 19: 448-457, doi: 10.1038/sj.ijir.3901552.

10. Auyeung TW, Lee JSW, Kwok T, Leung J, Ohlsson C, Vandenput $L$, et al. Testosterone but not estradiol level is positively related to muscle strength and physical performance independent of muscle mass: A cross-sectional study in 1489 older men. Euro J Endocrinol 2011; 164: 811-817, doi: 10.1530/EJE-10-0952.

11. Sinha-hikim I, Roth SM, Lee MI, Bhasin S. Testosteroneinduced muscle hypertrophy is associated with an increase in satellite cell number in healthy, young men. Am J Physiol Endocrinol Metab 2003; 285: E197-E205, doi: 10.1152/ ajpendo.00370.2002.

12. Malkin CJ, Pugh PJ, Jones RD, Kapoor D, Channer KS, Jones TH. The Effect of Testosterone Replacement on Endogenous Inflammatory Cytokines and Lipid Profiles in Hypogonadal Men. J Clin Endocrinol Metab 2004; 89: 3313-3318, doi: 10.1210/jc.2003-031069.

13. Visser M, Pahor M, Taaffe DR, Goodpaster BH, Simonsick $E M, N e w m a n A B$, et al. Relationship of interleukin- 6 and tumor necrosis factor-alpha with muscle mass and muscle strength in elderly men and women: the Health $A B C$ Study. J Gerontol A Biol Sci Med Sci 2002; 57: M326-M332, doi: 10.1093/gerona/57.5.M326.

14. Ferreira JP, Sartor CD, Leal AM, Sacco IC, Sato TO, Ribeiro $\mathrm{IL}$, et al. The effect of peripheral neuropathy on lower limb muscle strength in diabetic individuals. Clin Biomech 2017; 43: 67-73, doi: 10.1016/j.clinbiomech.2017.02.003.

15. de Carvalho Froufe Andrade AC, Caserotti $P$, de Carvalho CM, de Azevedo Abade EA, da Eira Sampaio AJ. Reliability of concentric, eccentric and isometric knee extension and flexion when using the REV9000 Isokinetic Dynamometer. J Hum Kinet 2013; 37: 47-53.

16. Bornstein S, Rutkowski H, Vrezas I. Cytokines and steroidogenesis. Mol Cel Endocrinol 2004; 215: 135-141, doi: 10.1016/ j.mce.2003.11.022.

17. Park S, Goodpaster B, Strotmeyer E, Kuller L, Broudeau R, Kammerer $\mathrm{C}$, et al. Accelerated loss of skeletal muscle strength in older adults with type 2 diabetes the health, aging, and body composition study. Diabetes Care 2007; 30: 1507-1512, doi: $10.2337 / \mathrm{dc06}-2537$.

18. Schaap LA, Pluijm SMF, Deeg DJH, Penninx BW, Nicklas $B J$, Lips $P$, et al. Low testosterone levels and decline in physical performance and muscle strength in older men: findings from two prospective cohort studies. Clin Endocrinol 2008; 68: 42-50, doi: 10.1111/j.1365-2265.2007. 02997.x.
19. American Diabetes Association. Standards of medical care in diabetes. In: Diabetes by region [Internet]. 2014. p. 86-89. Available from: http://www.cabi.org/cabebooks/ebook/20073 012958

20. Bhasin S, Cunningham GR, Hayes FJ, Matsumoto AM, Snyder PJ, Swerdloff RS, et al. Testosterone therapy in men with androgen deficiency syndromes: An endocrine society clinical practice guideline. J Clin Endocrinol Metab 2010; 95: 2536-2559, doi: 10.1210/jc.2009-2354.

21. Bakker K, Apelqvist J, Schaper NC. Practical guidelines on the management and prevention of the diabetic foot 2011. Diabetes Metab Res Rev 2012; 28: 225-231, doi: 10.1002/ dmrr.2253.

22. Watari R, Sartor CD, Picon AP, Butugan MK, Amorim CF, Ortega NRS, et al. Effect of diabetic neuropathy severity classified by a fuzzy model in muscle dynamics during gait. J Neuroeng Rehab 2014; 11: 11, doi: 10.1186/1743-0003-11-11.

23. Picon AP, Ortega NRS, Watari $R$, Sartor $C$, Sacco ICN. Classification of the severity of diabetic neuropathy: a new approach taking uncertainties into account using fuzzy logic. Clinics (São Paulo) 2012; 67: 151-156, doi: 10.6061/clinics/ 2012(02)10.

24. Furuyama S, Mayes DM; Nugentc CA. A radioimmunoassay for plasma testosterone. Steroids 1970; 16: 415-428, doi: 10.1016/S0039-128X(70)80124-6.

25. Research Reagents and Assays / Immunoassays / ELISA / ELISA Kits Instruction Manual BD OptEIA ${ }^{\mathrm{TM}}$ [Internet]. Available from: http://www.bdbiosciences.com/nvCategory.jsp?action= SELECT\&form=formTree_catBean\&item $=745107$

26. Cohen J. Statistical power analysis for the behavioral sciences [Internet]. Academic Press; 1977 [cited 2017 Mar 23]. 474 p. Available from: http://www.sciencedirect.com/science/book/ 9780121790608

27. Stigler SM. Francis Galton's Account of the Invention of Correlation. Statistical Science 1989; 4: 73-79, doi: 10.1214/ss/ 1177012580.

28. Hatef B, Bahrpeyma F, Mohajeri Tehrani MR. The comparison of muscle strength and short-term endurance in the different periods of type 2 diabetes. J Diabetes Metabc Disord 2014; 13 : 22, doi: 10.1186/2251-6581-13-22.

29. Andreassen CS, Jensen JM, Jakobsen J, Ulhøj BP, Andersen $\mathrm{H}$. Striated muscle fiber size, composition, and capillary density in diabetes in relation to neuropathy and muscle strength. J Diabetes. 2014; 6: 462-471, doi: 10.1111/1753-0407.12124.

30. Roy TA, Blackman MR, Harman SM, Tobin JD, Schrager M, Metter EJ. Interrelationships of serum testosterone and free testosterone index with FFM and strength in aging men. Am J Physiol Endocrinol Metab 2002; 283: E284-E294, doi: 10.1152/ajpendo.00334.2001.

31. Wang $\mathrm{X}, \mathrm{Hu} \mathrm{Z}, \mathrm{Hu} \mathrm{J}$, Du J, Mitch WE. Insulin resistance accelerates muscle protein degradation: Activation of the ubiquitin-proteasome pathway by defects in muscle cell signaling. Endocrinology 2006; 147: 4160-4168, doi: 10.1210/ en.2006-0251.

32. Kelley DE, He J, Menshikova E V, Ritov VB. Dysfunction of mitochondria in human skeletal muscle in type 2 diabetes. Diabetes 2002;51: 2944-2950, doi: 10.2337/diabetes.51. 10.2944.

33. Johnson ML, Robinson MM, Nair KS. Skeletal muscle aging and the mitochondrion. Trends Endocrinol Metab 2013; 24: 247-256, doi: 10.1016/j.tem.2012.12.003. 
34. Cesari M, Penninx BW, Pahor M, Lauretani F, Corsi AM, Rhys Williams G, et al. Inflammatory markers and physical performance in older persons: the InCHIANTI study. J Gerontol A Biol Sci Med Sci 2004; 59: 242-248, doi: 10.1093/gerona/59.3.M242.

35. Powers SK, Kavazis AN, Mcclung JM. Free radical biology in skeletal muscle. Oxidative stress and disuse muscle atrophy. J Appl Physiol 2007; 102: 2389-2397, doi: 10.1152/ japplphysiol.01202.2006
36. Glass DJ. Skeletal muscle hypertrophy and atrophy signaling pathways. Int J Biochem Cell Biol 2005; 37: 1974-1984, doi: 10.1016/j.biocel.2005.04.018.

37. Sandri M. Signaling in muscle atrophy and hypertrophy. Physiology 2008; 23: 160-170, doi: 10.1152/physiol.00041.2007.

38. Marzetti E, Calvani R, Bernabei R, Leeuwenburgh C. Apoptosis in skeletal myocytes: a potential target for interventions against sarcopenia and physical frailty - a mini-review. Gerontology 2012; 58: 99-106, doi: 10.1159/000330064. 